ESJ Humanities

\title{
Exploitation de l'espece Anadara Senilis (Arches) dans le Parc National de la Langue de Barbarie (PNLB), Gandiol (Senegal)
}

\author{
El Hadji Sow \\ Laboratoire Leïdi « Dynamiques des Territoires et Développement», \\ Université Gaston Berger de Saint-Louis, Sénégal, UFR des lettres et \\ sciences humaines, Centre de Suivi Écologique (CSE), Rue Léon Gontran \\ Damas, Fann Résidence-Dakar, Sénégal \\ Ousseynou Coly \\ Papa Abdoulaye Ndiaye \\ Cheikh Niang \\ Laboratoire Leïdi « Dynamiques des Territoires et Développement», \\ Université Gaston Berger de Saint-Louis, UFR des lettres et sciences \\ humaines, Sénégal
}

Doi:10.19044/esj.2021.v17n39p117

Submitted: 08 September 2021

Accepted: 22 November 2021

Published: 30 November 2021
Copyright 2021 Author(s)

Under Creative Commons BY-NC-ND

4.0 OPEN ACCESS

Cite As:

Sow E.H., Coly O., Ndiaye P.A. \& Niang C. (2021). Exploitation de l'espece Anadara Senilis (Arches) dans le Parc National de la Langue de Barbarie (PNLB), Gandiol (Senegal). European Scientific Journal, ESJ, 17 (39), 117.

https://doi.org/10.19044/esj.2021.v17n39p117

\section{Resumé}

Dans l'espace du Gandiol, les activités économiques traditionnelles étaient la pêche et l'agriculture avec lesquelles la population a une longue tradition. En 2003, les fortes pluies enregistrées dans le Haut bassin du fleuve Sénégal expliquent l'augmentation massive du débit $\mathrm{du}$ fleuve consécutivement à l'addition des ondes de crue. Pour empêcher des inondations dans la ville de Saint-Louis, une brèche a été ouverte. Cependant, cette brèche a eu des effets négatifs sur l'agriculture et beaucoup d'espèces de poissons. Elle a, aussi, entrainé des modifications écologiques se manifestant par l'apparition de certaines espèces aquatiques : Anadara Senilis, Carasostrea Gasar. Dès lors, l'étude de l'exploitation de l'espèce Anadara senilis dans le parc national de la langue de Barbarie (commune de Ndiebène Gandiol) fait l'objet de cette contribution. La méthodologie adoptée intègre les enquêtes de terrain sous forme de questionnaires et de guides d'entretien 
et le traitement des données quantitatives et qualitatives. Les résultats montrent que la collecte des coquillages est progressivement devenue la principale activité des femmes. Cette activité est pratiquée dans un but essentiellement lucratif et a eu des impacts considérables tant au niveau socioéconomique qu'environnemental. C'est pourquoi, l'encadrement des exploitants est souhaitable pour une gestion durable de cette ressource renouvelable.

Mots cles: Anadara senilis, Gandiol, coquillages, exploitation, principale ressource, brèche

\title{
Exploitation of Arches (Anadara Senilis) in the National Park of the Langue of Barbarie, Gandiol, Senegal
}

\begin{abstract}
El Hadji Sow
Laboratoire Leïdi « Dynamiques des Territoires et Développement », Université Gaston Berger de Saint-Louis, Sénégal, UFR des lettres et sciences humaines, Centre de Suivi Écologique (CSE), Rue Léon Gontran Damas, Fann Résidence-Dakar, Sénégal

Ousseynou Coly

Papa Abdoulaye Ndiaye

Cheikh Niang

Laboratoire Leïdi « Dynamiques des Territoires et Développement », Université Gaston Berger de Saint-Louis, UFR des lettres et sciences humaines, Sénégal
\end{abstract}

\begin{abstract}
In the Gandiol area, the traditional economic activities were fishing and agriculture, with which the population has a long tradition. In 2003, the heavy rains recorded in the upper Senegal River basin explain the massive increase in the river's flow following the addition of flood waves. To prevent flooding in the city of Saint-Louis, a breach was opened. However, this breach had negative effects on agriculture and many fish species. It has also caused ecological changes manifested by the appearance of certain aquatic species: Anadara Senilis, Carasostrea Gasar. Therefore, the study of the exploitation of the species Anadara senilis in the National Park of the Langue de Barbarie (municipality of Ndiebène Gandiol) is the subject of this contribution. The methodology adopted integrates field surveys in the form of questionnaires and interview guides and the processing of quantitative and qualitative data. The results show that shellfish collection has gradually become the main activity of women. This activity is practiced essentially for profit and has had
\end{abstract}


considerable impacts at both the socio-economic and environmental levels. This is why the supervision of the operators is desirable for a sustainable management of this renewable resource.

Keywords: Anadara senilis, Gandiol, shellfish, exploitation, main resource, breach

\section{Introduction}

Le Gandiol est à cheval entre les Niayes du littoral Nord sénégalais et le delta du fleuve Sénégal (Guèye, 2012). C'est un territoire qui se situe en aval du barrage de Diama, au sud de la ville de Saint-Louis. Situé à $16^{\circ} 15^{\prime}$ de Latitude Nord et $16^{\circ} 25^{\prime}$ de Longitude Ouest, le Gandiol jouxte la commune de Saint-Louis, à l'est, par la commune de Fass Ngom, la RN2 et la zone du Toubé, au sud, par la commune rurale de Léona dans le département de Louga. Il s'insère entre le fleuve Sénégal et l'Océan atlantique, à l'ouest, soit une superficie d'environ $360 \mathrm{~km}^{2}$ (figures 1 et 2).

Le Gandiol bénéficiait, avant le colmatage de l'ancienne embouchure, des derniers kilomètres parcourus par les eaux du fleuve Sénégal, après un trajet de près de $1800 \mathrm{~km}$. Il est caractérisé par l'alternance de deux saisons annuelles : une saison humide de juillet à septembre et une saison sèche de novembre à juin. Les pluies sont enregistrées durant l'hivernage avec la présence de la mousson en provenance du Sud, issue de l'anticyclone de Sainte-Hélène. Elles sont peu abondantes et dépassent rarement $350 \mathrm{~mm}$ par an dans le Nord des Niayes. Des pluies appelées heug ou «pluies des mangues », surviennent souvent en saison sèche, notamment de décembre à février (saison fraiche intermédiaire). Ces précipitations issues d'intrusion de masses d'air polaire, irrégulières et peu abondantes, sont cependant d'une grande importance pour la pratique des cultures de contre-saison dans ce milieu (Jacoutot, 2006). Le relief, plat dans l'ensemble, est marqué par les variations du niveau marin durant le quaternaire et l'évolution du fleuve, qui ont entraîné l'édification des systèmes dunaires notamment celui dit ogolien (22 000 - 12 000 ans BP) et la formation des sols hérités du pluvial Tchadien (12 $000-8$ 000 ans $B P$ ). Ainsi, il est marqué par une succession de dunes littorales (blanches et jaunes) et continentales (rouges) mais aussi de dépressions interdunaires (Niayes et Ndioukis). Sous ces unités morphopédologiques se localisent une nappe phréatique affleurante à sub-affleurante dont la qualité chimique varie suivant les secteurs. Ce contexte justifiait les activités axées essentiellement sur le maraichage, la pêche artisanale, l'élevage de petits ruminants, etc. 


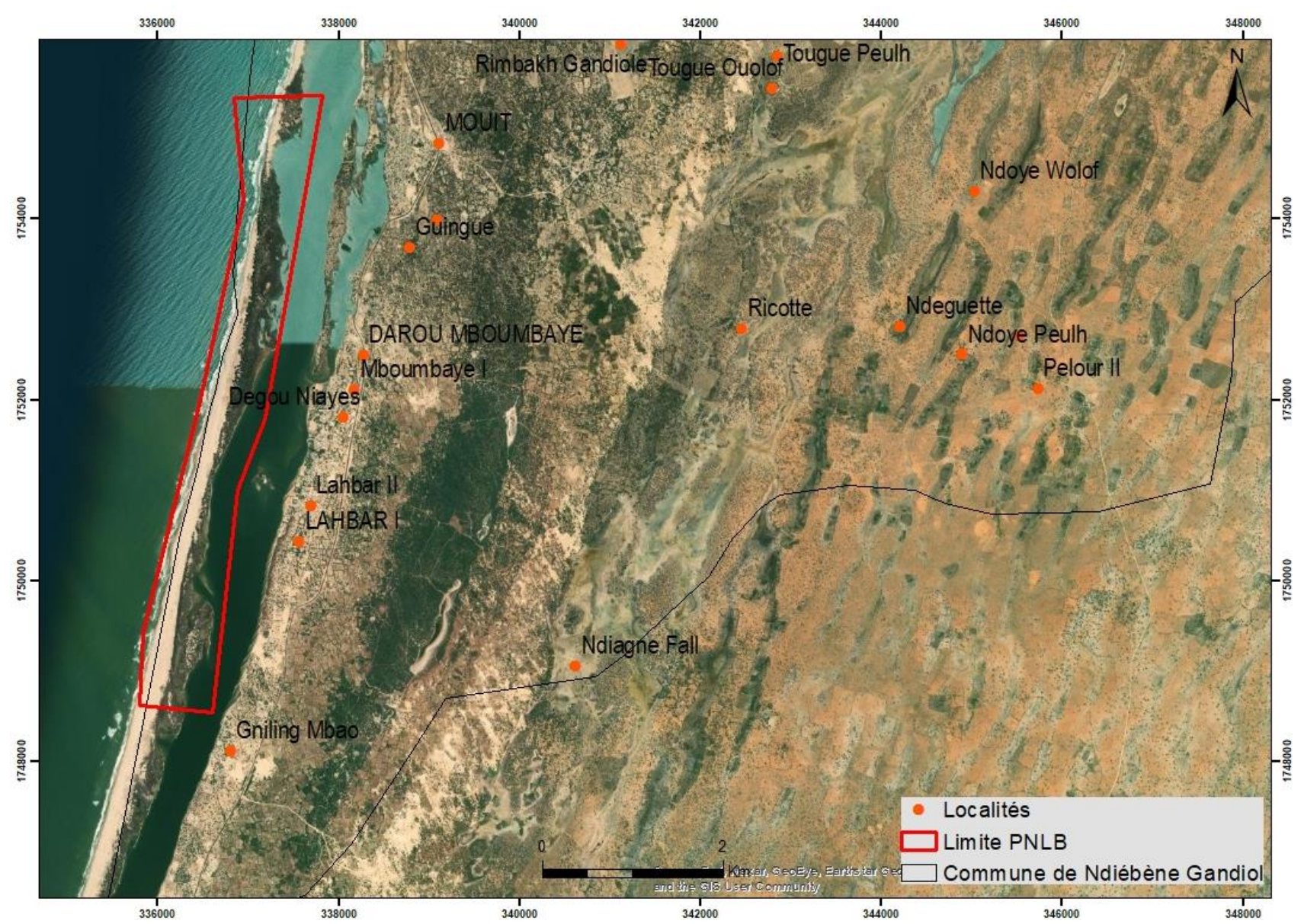

Figure 1. Spatio carte de la commune de Ndiébène Gandiol et du parc national de la langue de Barbarie

Durant la période coloniale, le Gandiol produisait diverses denrées destinées à la population européenne qui vivait à Saint-Louis : la patate douce, le manioc, le haricot, le niébé, le béréf, la pomme de terre, le piment, la tomate. Les produits étaient facilement écoulés sur les marchés de la capitale coloniale (Saint-Louis). A côté de l'activité agricole se développaient la pêche maritime et continentale, l'extraction du sel dans les anciens golfes nouakchottiens et l'activité pastorale. Cependant la sécheresse des années 1970 et la mise en service des deux barrages sur le fleuve Sénégal, particulièrement celui de Diama vont entrainer des modifications du milieu naturel du Gandiol (Jacoutot, 2006). Ces modifications ont favorisé le développement du maraichage qui connait une expansion jusqu'au début des années 2000.

En 2003, les fortes pluviométries enregistrées dans le Haut bassin du Sénégal ( $812 \mathrm{~mm}$ en 2003 contre $585 \mathrm{~mm}$ en 2002 et $488 \mathrm{~mm}$ en 2001) ont engendré l'augmentation exponentielle du débit du fleuve, passant de 1000 
$\mathrm{m}^{3} / \mathrm{s}$ à $10000 \mathrm{~m}^{3} / \mathrm{s}$. Ces ondes de crue menaçaient d'inonder la ville de SaintLouis (particulièrement le quartier de Sor). Pour éviter cela, les autorités vont, dans la nuit du 3 au 4 octobre 2003, creuser un canal de délestage sur la Langue de Barbarie afin de sauver la ville (Sy B. A. et Sy A. A., 2010).

Toutefois, cette brèche ne cesse depuis lors de s'étendre atteignant environ $5000 \mathrm{~m}$ de largeur en 2014. Elle a eu des effets négatifs sur toutes les activités en général et particulièrement sur l'agriculture et la pêche à travers l'augmentation de la salinisation des terres et la baisse du $\mathrm{pH}$ de l'eau avec l'intrusion massive des eaux de la mer consécutive à la l'ouverture de la brèche sur la Langue de Barbarie. Beaucoup d'espèces de poissons disparaissent, l'agriculture sous pluie diminue de manière très sensible parallèlement à la migration du front maraicher vers le sud. Cet aménagement a, en outre, entrainé des modifications écologiques de plus en plus incontrôlables, se manifestant par des inconvénients et des opportunités économiques notamment l'apparition de certaines espèces aquatiques : Anadara senilis, Carasostrea gasar. L'exploitation de ces coquillages est devenue une activité génératrice de revenus dans le Gandiolais et particulièrement dans le parc national de la Langue de Barbarie. 


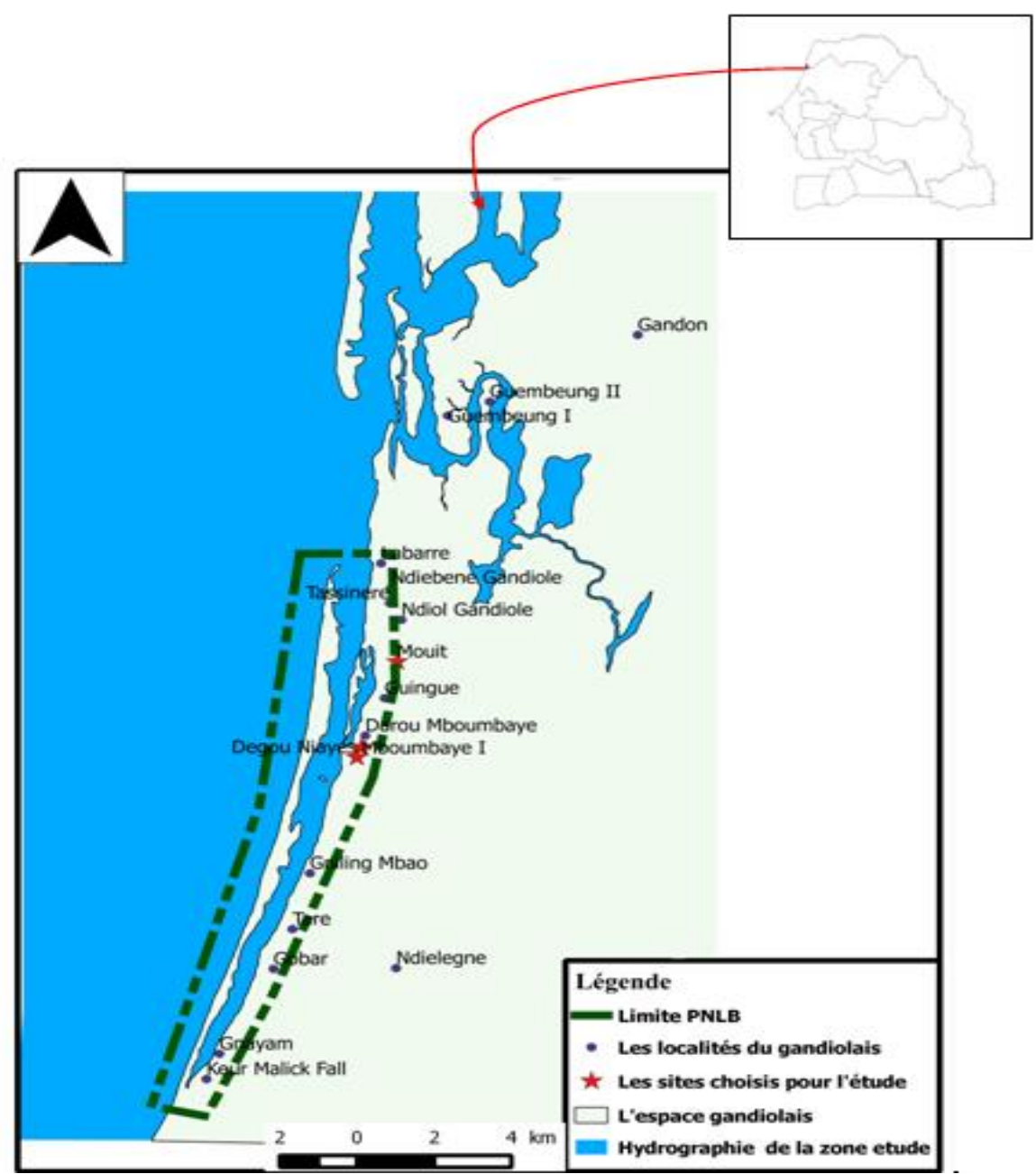

Figure 2 : Localisation du Gandiolais et du Parc National de la Langue de Barbarie

Cette activité d'exploitation des coquillages apparait notamment avec l'élargissement de la brèche qui a entrainé la diminution de l'effet de chasse des courants, donc une accumulation plus importante des sédiments dans le chenal fluvial. Elle constitue l'activité quotidienne de beaucoup des femmes des villages du Gandiol : Mouit, Mboumbaye, Dègou Niaye. C'est une source de revenus importante pour les ménages déjà durement éprouvés par les conséquences néfastes de la brèche avec le déclin de l'agriculture de décrue, chute de la production de rente, etc. Cependant cette exploitation échappe au contrôle des services étatiques et demeure toujours une activité informelle, ce qui justifie la nécessité d'évaluer cette nouvelle activité génératrice de revenus (AGR) à travers la dynamique organisationnelle des acteurs, les flux commerciaux et les impacts socio-économiques et environnementaux. Pour répondre à ces attentes de recherches, une méthodologie a été appliquée. 


\section{Données et méthodes}

La méthodologie comporte la collecte et le traitement des données (tableau 1). Le principal logiciel utilisé est le logiciel sphinx. En effet, de la conception du questionnaire à la présentation des conclusions de l'étude, le SPHINX met à la disposition du chercheur un ensemble intégré de ressources utiles pour la conception et la réalisation du questionnaire, la saisie des réponses, le dépouillement, l'analyse et la présentation des résultats (Moscarola J. 1989).

\begin{tabular}{|c|c|c|}
\hline ETAPES & ACTIVITES & MATERIELS/OUTILS \\
\hline $\begin{array}{l}\text { Collecte des } \\
\text { données }\end{array}$ & $\begin{array}{l}\text { - Activité exploratoire de l'espace } \\
\text { d'exploitation et prise de contact avec } \\
\text { les services du PNLB } \\
\text { - } \quad \text { recensement des sites d'exploitation } \\
\text { - } \quad \text { enquête auprès des acteurs (réseaux de } \\
\text { collecte et de transformation) } \\
\text { - entretien avec le service de pêche, des } \\
\text { eaux et forêt, les autorités locales } \\
\text { - visite des points de vente } \\
\text { - rencontre avec les commerçants des } \\
\text { - coquillages } \\
\text { enquête auprès des exploitants }\end{array}$ & $\begin{array}{ll}\text { - } & \text { questionnaire } \\
\text { - } & \text { guide } \\
& \text { d'entretien } \\
\text { - } & \text { enregistreur } \\
\text { - } & \text { appareils photo } \\
\text { - } & \text { GPS }\end{array}$ \\
\hline $\begin{array}{l}\text { Traitement des } \\
\text { données }\end{array}$ & $\begin{array}{l}\text { - } \quad \text { analyse de la dynamique } \\
\text { organisationnelle } \\
\text { - } \quad \text { analyse des flux commerciaux } \\
\text { - évaluation et analyse des retombés } \\
\text { économiques et leurs impacts }\end{array}$ & $\begin{array}{ll}\text { - } & \text { Word, excel, sphinx, } \\
\text { - } & \text { Logiciels } \\
\text { cartographiques } \\
\text { (global Mapper, } \\
\text { Google earth, Qgis). }\end{array}$ \\
\hline
\end{tabular}

Tableau 1 : Récapitulatif de la méthodologie du travail

L'échantillonnage : il porte sur les personnes qui ont été interrogées dans les villages ciblés (Mouit, Mboumbaye, Dègou Niaye). L'échantillonnage utilisé est une méthode non aléatoire appelée l'échantillonnage au jugé. Elle consiste à prendre les unités de l'échantillon parmi celles que l'on considère des unités typiques de la population (Paillé, 2006). Dans le cas de cette enquête auprès des populations locales, les personnes qui s'activent dans l'exploitation des coquillages ont été privilégiées car elles sont plus aptes à expliquer la dynamique de cette activité de par leur expérience. Ceci pour éviter la collecte de données erronées. Dans chaque village, un certain nombre de personnes ont été interrogées. Aussi, comme la population totale de chaque village est connue, le pourcentage des personnes interrogées dans chacun de ces 03 villages a été calculé. La méthode de calcul de ces pourcentages s'est faite de la manière suivante : 
$\mathbf{N}=$ population totale du village ciblé

$\mathbf{n}=$ taille de l'échantillon du village ciblé (nombre de personnes interrogées)

$\mathbf{P} \%=$ pourcentage des personnes interrogées du village ciblé

Formule P \% $=(\mathrm{n} \times 100 \%) / \mathrm{N}$

- Pourcentage des personnes interrogées dans le village de Mouit : $\mathrm{P} \%=(30$ x 100) / $1391=2,1 \%$

- Pourcentage des personnes interrogées dans le village de Mboumbaye : P \% $=(9 \times 100) / 428=2,1 \%$

- Pourcentage des personnes interrogées dans le village de Dègou Niaye : $\mathrm{P} \%$ $=(11 \times 100) / 803=1,36 \%$

\section{Résultats}

L'ensemble des données collectées et des méthodes appliquées ont abouti aux résultats ci-dessous.

\subsection{Dynamique organisationnelle de la collecte des coquillagess}

L'apparition des coquillages dans les villages de Mouit, de Mboumbaye et de Dègou Niaye remonte en 2008. Pratiquée depuis 2011 dans le secteur, le ramassage des coquillages est progressivement devenu la principale activité des femmes. La tranche d'âge des exploitants varie de 15 à 58 ans.

Le nombre d'exploitants varie en fonction du lieu et des saisons. En effet, quelque 9,8\% des exploitants s'adonnent à cette activité pendant la saison chaude ${ }^{1} ; 25,5 \%$ pendant la saison des pluies $^{2} ; 9,8 \%$ pendant la saison sèche $^{3}$ et $54,9 \%$ pendant toute l'année. Le nul statistique ne signifie pas forcément l'absence d'exploitation pendant la saison froide mais démontre qu'il n'y a pas d'exploitants qui s'y adonnent de manière exclusive (figure 3).

${ }^{1}$ Saison chaude : 09 mois (de mars à novembre)

${ }^{2}$ Saison des pluies : 04 mois (de juillet à octobre)

${ }^{3}$ Saison sèche : 08 mois (de novembre à juin) 


\begin{tabular}{|c|c|c|c|}
\hline \multicolumn{4}{|c|}{ Période de collecte } \\
\hline & $\mathrm{Nb}$ & $\%$ cit. & \\
\hline période chaude & 5 & $9,8 \%$ & $9,8 \%$ \\
\hline période froide & $\mathbf{0}$ & $0,0 \%$ & $0,0 \%$ \\
\hline saison séche & 5 & $9,8 \%$ & $9,8 \%$ \\
\hline saison des pluies & 13 & $25,5 \%$ & $25,5 \%$ \\
\hline toute l'année & 28 & $54,9 \%$ & $54,9 \%$ \\
\hline Total & 51 & $100,0 \%$ & \\
\hline
\end{tabular}

Figure 3 : Saisons de ramassage des coquillages

Pour le cas de Mouit, selon les enquêtes et les entretiens, la collecte est faite par les hommes avec une technique de dragage. Cette dernière permet de récolter les coquillages en attachant le dispositif constitué d'un anneau et d'un filet pour collecter les arches au fond de l'eau (photo 1).

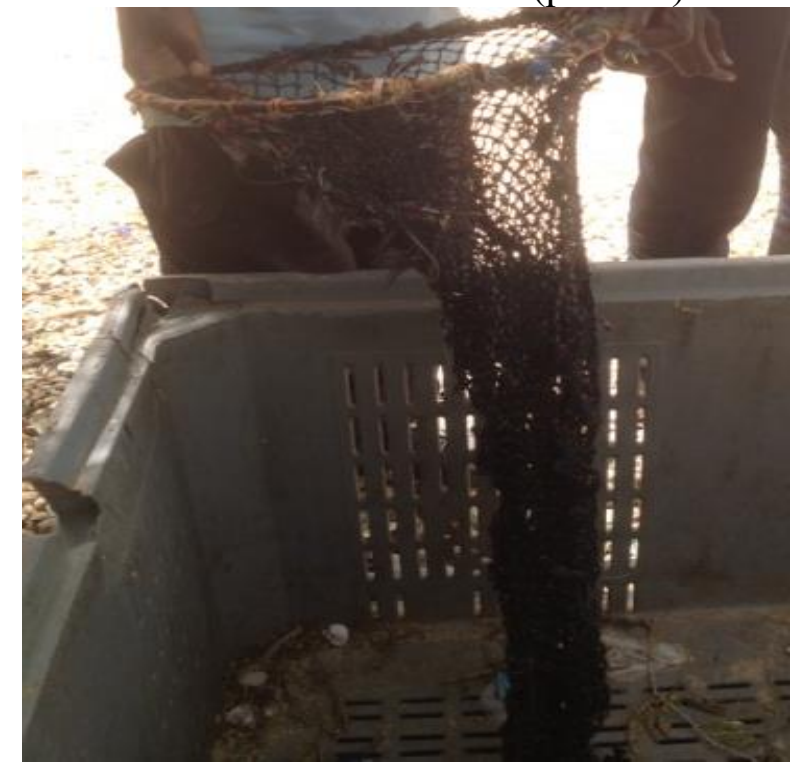

Photo 1 : Dispositif de collecte des arches utilisé à Mouit

L'usage de ce dispositif de collecte s'explique par la profondeur qui empêche le ramassage à la main. La taille des coquillages collectés dans cette localité varie entre 4,5 et $6 \mathrm{~cm}$ (photo 2). 


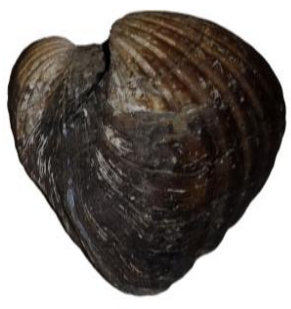

1
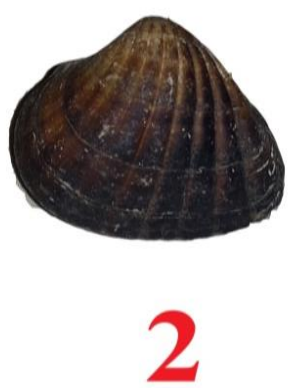
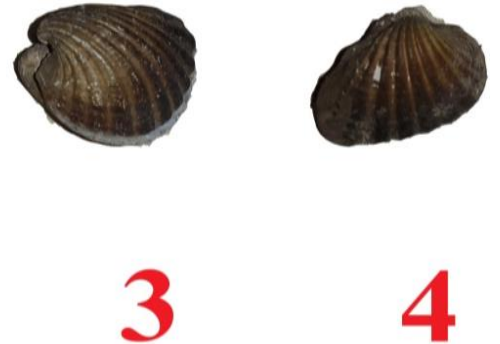

Photo 2 : Taille des coquillages selon la localité

1. $6 \mathrm{~cm}$ (prélevé à Mouit)

2. $4,5 \mathrm{~cm}$ (prélevé à Mouit, Mboumbaye et Dègou Niaye)

3. $3 \mathrm{~cm}$ (prélevé à Mboumbaye et Dègou Niaye)

4. 2,5 cm (prélevé à Mboumbaye et Dègou Niaye)

Ces coquillages (non transformés) sont ensuite vendus aux femmes à 1000f CFA la bassine. Dans le secteur de Mouit, la durée de collecte est de 2 mois. Cette séquence coïncide avec le retour des pêcheurs ${ }^{4}$ et/ ou les vacances scolaires, ce qui favorise la disponibilité de moyens et de mains d'œuvre pour la collecte. Pour les 10 autres mois, la majeure partie de ces femmes se rendent à Mboumbaye distant de $3 \mathrm{~km}$ pour y pratiquer cette activité. Cependant, il faut noter qu'à Mouit, cette collecte est devenue de moins en moins pratiquée comparée aux deux autres villages (Mboumbaye et Dègou Niaye) où la collecte se fait à marée basse essentiellement par les femmes et surtout les adultes (photo 3 ).

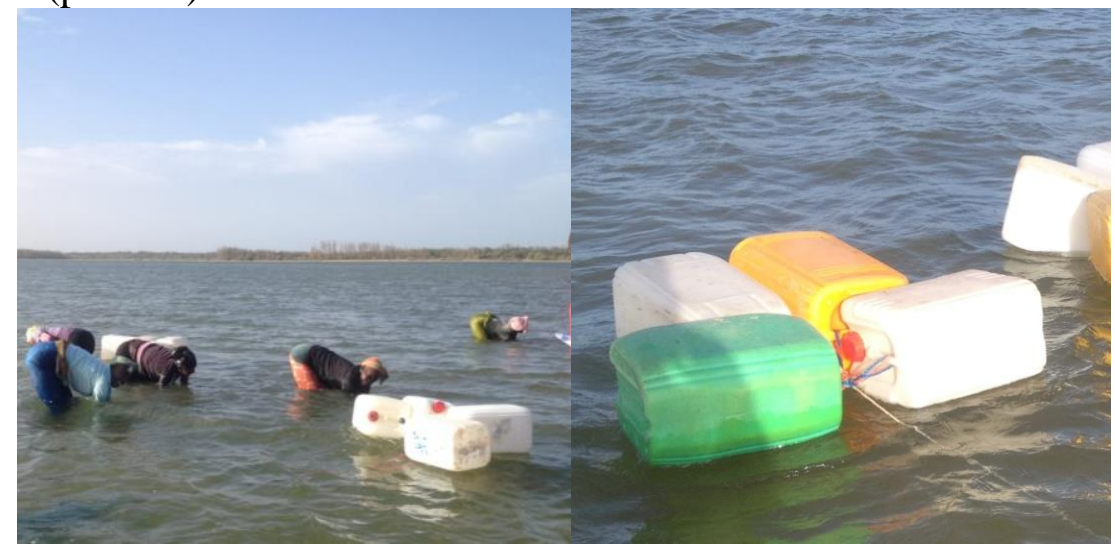

Photo 3 : Collecte des arches à Mboumbaye

Cette collecte se fait à la main. La taille des coquillages ramassés dans les deux localités varie de 4,5 à 2,5 cm (photo 2). Pour transporter les sacs de

${ }^{4}$ Les pêcheurs qui suivaient les bancs des poissons reviennent dans leurs villages d'origine pendant les fêtes de tabaski. 
coquillages, les femmes utilisent un dispositif formé de 4 bidons vides de 20 litres reliés entre eux sous la forme d'une plate-forme mobile flottante sur laquelle la récolte est posée (photo 3 , image de droite).

Dans ces 2 villages, l'activité se fait durant toute l'année sauf pendant la saison froide et les marées hautes. La quantité journalière collectée est plus importante dans les villages de Mboumbaye et de Dègou Niaye comparés à Mouit. Autrement dit, dans les villages de Mboumbaye et de Dègou Niaye, la quantité maximale collectée par jour et par un exploitant est de $15 \mathrm{~kg}$ contre $10 \mathrm{~kg}$ à Mouit. Du point de vue organisationnel, le constat montre beaucoup de limites :

- les exploitants ne sont regroupés ni en coopérative, ni en GIE comme ça se fait dans d'autres localités comme Dionewar';

- la collecte de la ressource n'est pas sélective ;

- le repos biologique n'est pas observé ;

- le contrôle et l'appui financier et technique des autorités locales et des organismes ne sont pas assurés. Après la collecte, les arches sont transformées en 3 phases :

- phase de cuisson où les coquillages sont mis dans une marmite avec un peu d'eau, puis chauffés de 15 à $20 \mathrm{mn}$ (photo 4) ;

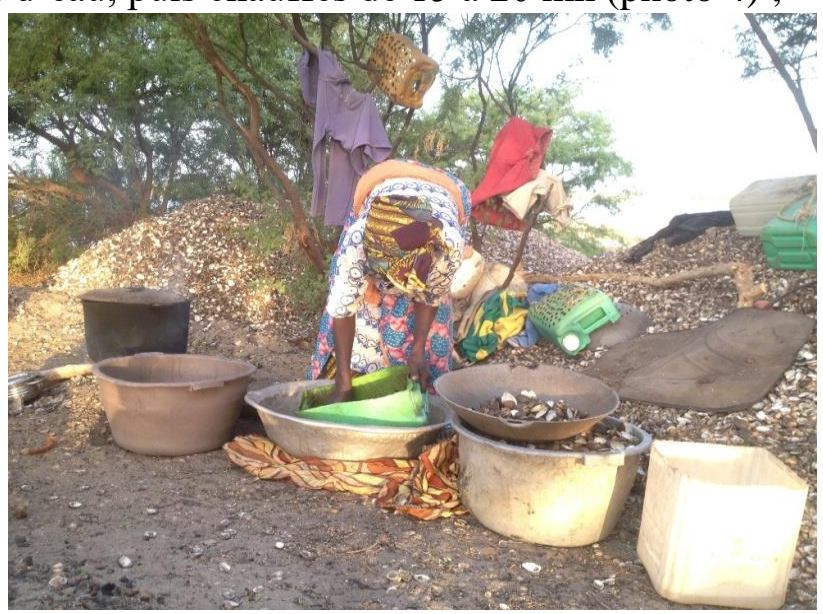

Photo 4 : Transformation des arches

- phase de décorticage où les coquillages sont versés dans un tamis et agités afin de séparer les arches de leurs coquilles (photo 5);

${ }^{5}$ Les exploitantes de Dionewar sont regroupées en GIE et bénéficient d'un appui technique, matériel et financier. La filière est ainsi bien organisée et aboutie à l'exportation du produit transformé à l'extérieur. 


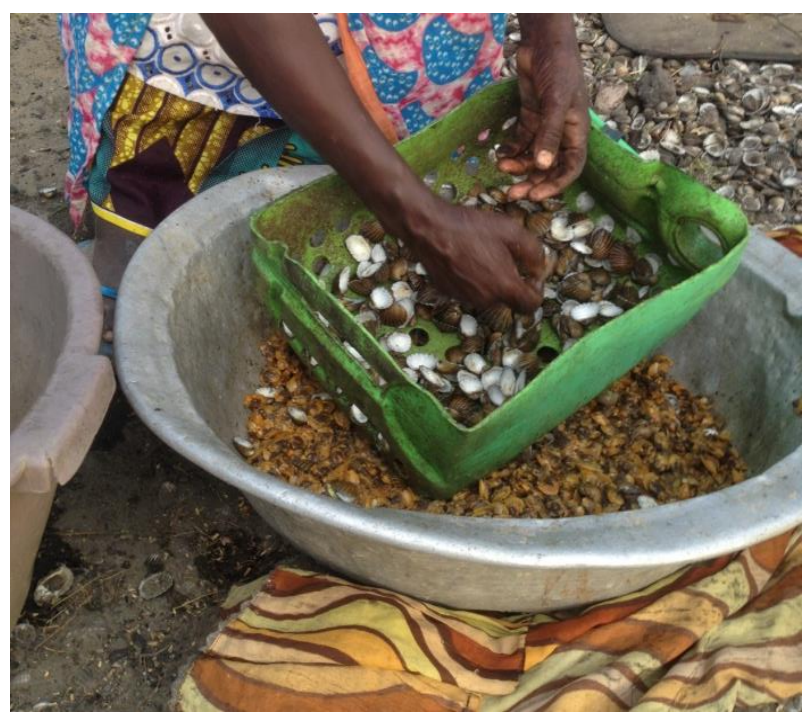

Photo 5 : Décorticage des arches

- phase de séchage où les arches sont versées sur un lit de séchage et exposés au soleil pour une durée de 24 à 48 heures (photo 6).

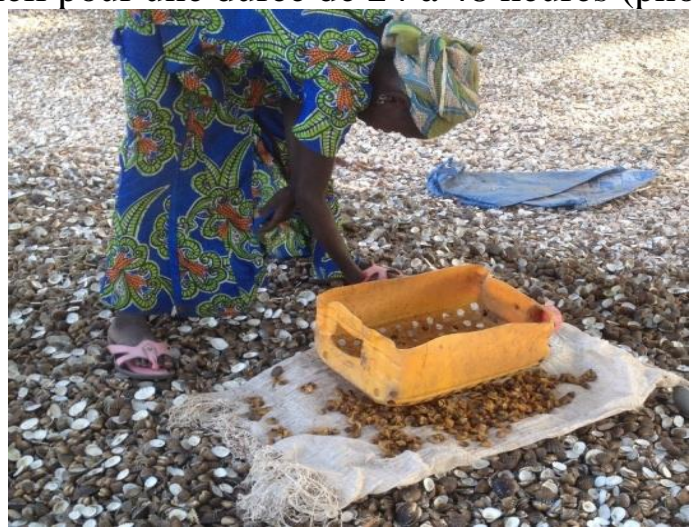

Photo 6 : Le séchage des arches

Il apparaît que le processus de transformation se fait d'une manière artisanale où le respect des règles d'hygiène est perfectible. C'est là une preuve que la collecte montre beaucoup de limites tant du point de vue organisationnel de la récolte que dans la chaine de transformation, ce qui ne constitue pas une contrainte de l'écoulement de l'ensemble du produit.

\subsection{Les flux commerciaux}

Les coquillages récoltés sont vendus dans des endroits différents et permettent de générer des revenus. 


\subsubsection{La quantité récoltée}

La quantité récoltée varie en fonction des saisons (figure 4).

\begin{tabular}{|c|c|c|c|c|c|c|c|}
\hline \multicolumn{8}{|c|}{ Quantité par rapport à la période } \\
\hline & $\begin{array}{l}\text { Moins } \\
\text { de } 4\end{array}$ & De 4 à 5 & De 6 à 7 & De 8 à 9 & $\begin{array}{c}\text { De } 10 \text { à } \\
11\end{array}$ & $\begin{array}{l}12 \text { et } \\
\text { plus }\end{array}$ & Total \\
\hline période chaude & 2 & 2 & $\mathbf{0}$ & $\mathbf{0}$ & $\mathbf{0}$ & 1 & 5 \\
\hline période froide & $\mathbf{0}$ & $\mathbf{0}$ & $\mathbf{0}$ & 0 & 0 & 0 & $\mathbf{0}$ \\
\hline saison séche & 3 & 1 & $\mathbf{0}$ & $\mathbf{0}$ & $\mathbf{0}$ & $\mathbf{0}$ & 4 \\
\hline saison des pluies & 4 & 4 & $\mathbf{0}$ & 2 & 3 & $\mathbf{0}$ & 13 \\
\hline toute l'année & 9 & 8 & $\mathbf{0}$ & 3 & 5 & 3 & 28 \\
\hline Total & 18 & 15 & 0 & 5 & 8 & 4 & 50 \\
\hline période chaude & & & & $40 \%$ & & 20 & $100,0 \%$ \\
\hline période froide & $100,0 \%$ & & & & & & \\
\hline saison séche & & & & & & $25 \%$ & $100,0 \%$ \\
\hline saison des pluies & & & $30,8 \%$ & & & $23,1 \%$ & $100,0 \%$ \\
\hline toute l'année & & & $28,6 \%$ & & 17 & 10,7 & $100,0 \%$ \\
\hline Total & & & & $\%$ & & $3 \%$ & $100,0 \%$ \\
\hline
\end{tabular}

Figure 4 : La quantité d'arches (en $\mathrm{kg}$ ) récoltée en fonction de la période

On note pendant la saison chaude $9,8 \%$ d'exploitants dont :

- $\quad 40 \%$ récoltent moins de $4 \mathrm{Kg} /$ jour ;

- $40 \%$ récoltent entre 4 et $5 \mathrm{Kg} / \mathrm{jour}$;

- $20 \%$ seuls récoltent plus de $12 \mathrm{Kg} /$ jour.

Pendant la saison sèche on note $9,8 \%$ d'exploitants dont :

- $75 \%$ récoltent moins de $4 \mathrm{Kg} /$ jour ;

- $25 \%$ récoltent de 04 à $05 \mathrm{Kg} /$ jour.

Durant la saison des pluies il y a $25,5 \%$ d'exploitants dont:

- $\quad 30,8 \%$ récoltent moins de $4 \mathrm{Kg} /$ jour ;

- $30,8 \%$ récoltent 4 à $5 \mathrm{Kg} /$ jour ;

- $\quad-15,4 \%$ récoltent 8 à $9 \mathrm{Kg} /$ jour ;

- $23,1 \%$ récoltent 10 à $11 \mathrm{Kg} /$ jour.

La majorité des exploitants, soit 54,9\% s'adonnent quotidiennement à cette activité pendant toute l'année et parmi eux :

- $\quad 32,1 \%$ récoltent moins de $4 \mathrm{Kg} /$ jour ;

- $\quad 30 \%$ récolent de 4 à $5 \mathrm{Kg} /$ jour ;

- $\quad 10,7 \%$ récoltent de 8 à $9 \mathrm{Kg} / \mathrm{jour}$;

- $16 \%$ récolent de 10 à $11 \mathrm{Kg} / \mathrm{jour}$; 
- seuls $8 \%$ récoltent $12 \mathrm{Kg} /$ jour et plus.

\subsubsection{La commercialisation}

La vente des produits transformés se fait à domicile ou au marché hebdomadaire du mercredi. Le prix d'un kilogramme d'arche varie entre 1000 f et 1300 f. Les acheteurs sont des «bana-banas ${ }^{6}$ » qui proviennent principalement de Gandiol. Ceux-ci revendent ces produits dans la ville de Saint-Louis, à Touba et à Dakar (figure 5).

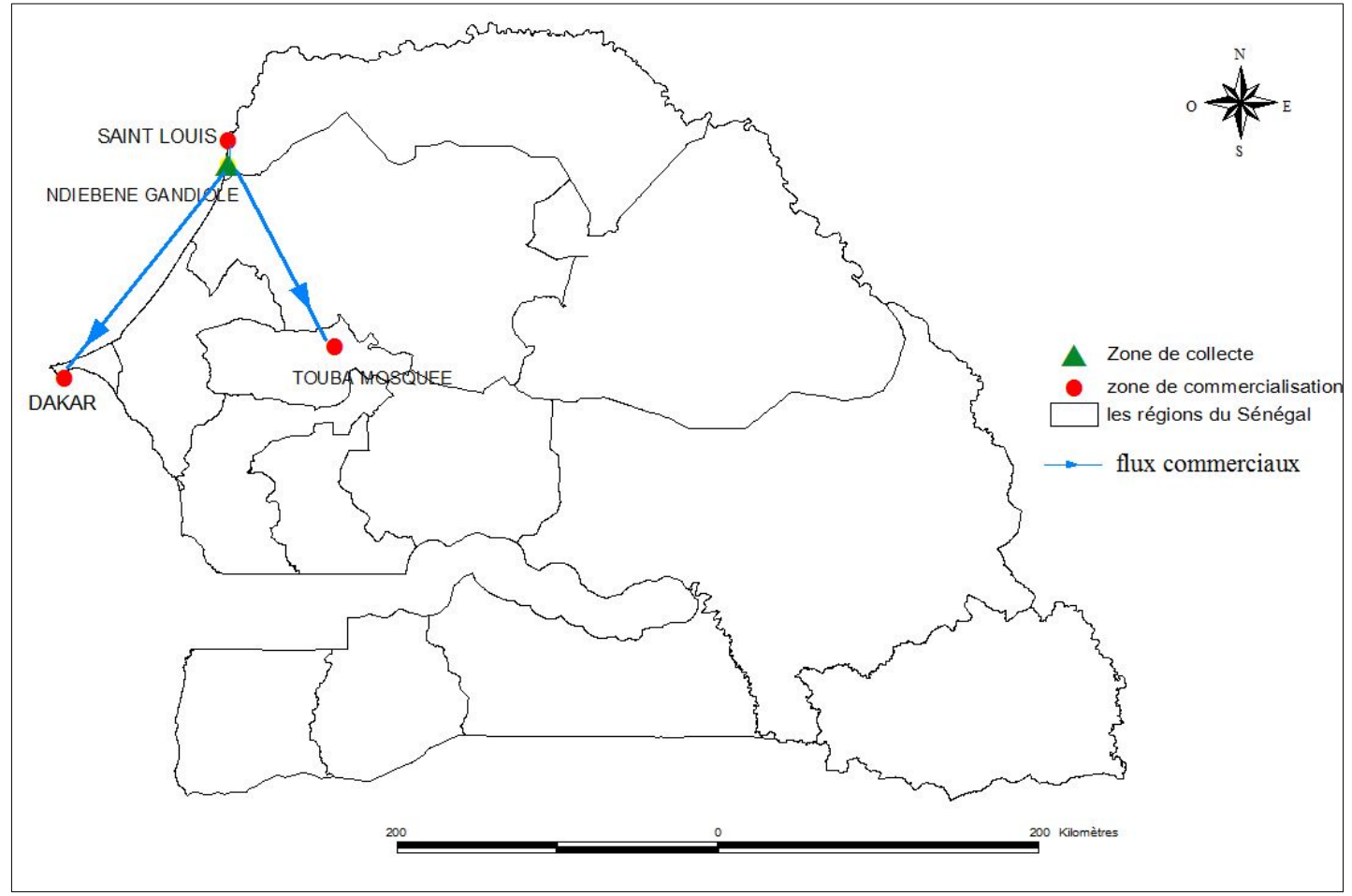

Figure 5 : Flux commerciaux

A Saint-Louis, les arches se vendent bien dans les marchés par kg et au détail. Le coût du $\mathrm{kg}$ varie de $2000 \mathrm{~F} \mathrm{CFA}$ à $2500 \mathrm{f} \mathrm{CFA}$. Les sachets sont vendus à 50f CFA l'unité (photo 7).

${ }^{6}$ Nom local qui signifie commerçant 


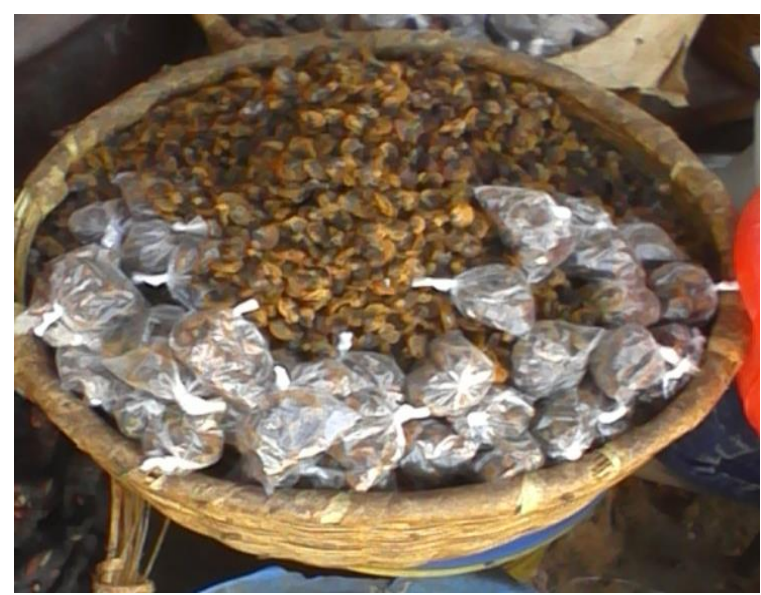

Photo 7 : Vente d'arche au marché de Saint Louis

Dans les hôtels par contre, les arches ne sont pas très prisées. Ceci s'explique par le fait que ce produit est méconnu de certains hôteliers selon un agent de l'hôtel SINDONE à Saint-Louis. En plus, le produit est jugé trop cher si on le compare aux poissons.

\subsection{Transformations socio-économiques et environnementaux}

Bien que récente dans la localité, l'activité de transformation des coquillages a entrainé des transformations positives au double plan socioéconomique et environnemental.

\subsubsection{Conséquences sur le plan socioéconomique}

La collecte des coquillages est l'activité la plus rentable ; les enquêtes révèlent que les revenus issus du commerce des arches sont plus importants que ceux de l'agriculture. Dans ce cadre, chaque exploitant récolte en moyenne 5,82 kg par jour, vendus à 7275 f CFA. Ces exploitants travaillent en moyenne 10 jours par mois, soit un profit moyen mensuel de $70450 \mathrm{f} \mathrm{CFA.}$ Certains exploitants gagnent mensuellement quelque $187500 \mathrm{f} \mathrm{CFA}$, ce qui correspond au revenu tiré d'une campagne agricole de 4 mois. 


\begin{tabular}{|c|c|c|c|}
\hline \multicolumn{4}{|c|}{ Profit Mensuel de l'exploitation } \\
\hline \multicolumn{3}{|c|}{$\begin{array}{l}\text { Moyenne }=70450,00 \\
\text { Médiane = } 50000,00 \\
\text { Min = } 25000 \quad \text { Max = } 187500\end{array}$} & \multirow[b]{3}{*}{$12,0 \%$} \\
\hline & $\mathrm{Nb}$ & $\%$ cit. & \\
\hline Moins de 30000 & 6 & $12,0 \%$ & \\
\hline De 30000 à 59999 & 27 & $54,0 \%$ & \multirow{3}{*}{$\begin{array}{l}\mid 2,0 \% \\
10,0 \%\end{array}$} \\
\hline De 60000 à 89999 & 1 & $2,0 \%$ & \\
\hline De 90000 à 119999 & 5 & $10,0 \%$ & \\
\hline De 120000 à 149999 & 7 & $14,0 \%$ & \multirow{3}{*}{\begin{tabular}{|l}
$14,0 \%$ \\
$8,0 \%$
\end{tabular}} \\
\hline 150000 et plus & 4 & $8,0 \%$ & \\
\hline Total & 50 & $100,0 \%$ & \\
\hline
\end{tabular}

Figure 6 : Revenu mensuel des exploitants (F CFA)

Au total, cette activité satisfait les besoins quotidiens des ménages. En outre, du point de vue social, elle diminue les charges financières des chefs de foyers mais aussi la dépendance vis-à-vis de l'extérieur ${ }^{7}$. Aussi, elle constitue un moyen pour les élèves de satisfaire leurs besoins notamment en frais scolaires et fournitures diverses. Il faut noter aussi que les revenus de l'activité de collecte des arches a permis à certains de transformer la morphologie de leur bâtis ${ }^{8}$.

Cependant, la collecte des arches entraine des effets négatifs car sur le plan sanitaire ; le contact prolongé avec l'eau froide peut entrainer le rhumatisme. A cela s'ajoute les risques de blessures et d'infections liés au non usage des gants et des chaussures adaptées lors de la collecte. Il faut aussi souligner les risques de noyades quand la collecte se déroule dans les poches profondes en sus du manque d'hygiène dans le processus de transformation :

- les marmites et les autres ustensiles utilisés peuvent être mieux nettoyés au préalable ;

- les séchages se font sur des nattes ou des moustiquaires à même le sol, exposés aux poussières ;

- le produit transformé n'est pas recouvert/protégé, ce qui peut entrainer des risques de contamination.

${ }^{7}$ Les revenus des émigrés sont souvent utilisés pour régler les besoins de leurs villages d'origine.

${ }^{8}$ Les bâtis qui étaient, jadis, sous forme de paille deviennent en durs. 


\subsubsection{Conséquences sur le plan environnemental}

L'activité de collecte des coquillages favorise l'idée de reboisement de la mangrove pour rendre le milieu de reproduction plus favorable et plus attractif qui pourrait se traduire par l'apparition et/ou le développement de nouvelles espèces aquatiques et une protection contre les agressions anthropiques :

- développement des huîtres ;

- l'avènement de nouvelles espèces de poissons (éthmaloge, etc.) ;

- lieu de reproduction et de développement de certains oiseaux d'eau ;

- réduction de la surexploitation des ressources ;

- revalorisation du parc national de la Langue de Barbarie.

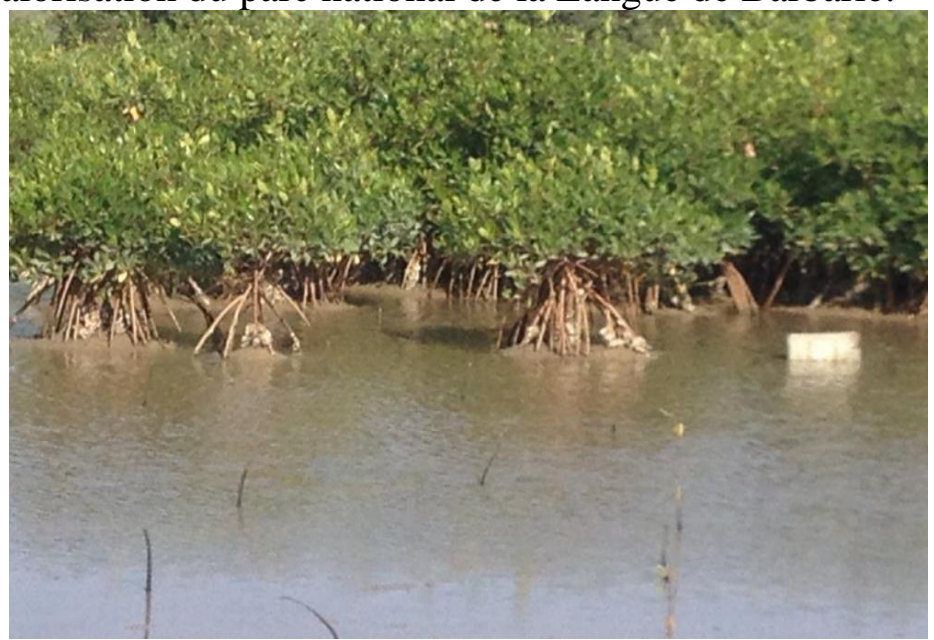

Photo 8 : Huitres colonisant les racines de la mangrove

Toutefois, le processus d'exploitation de la ressource doit être maitrisé car il masque beaucoup d'effets négatifs :

- risque de rareté des espèces exploitées consécutif au non-respect du repos biologique ${ }^{9}$;

- risque de rareté des espèces matures à cause du non pratique de la collecte sélective ;

- risque de transformation de la texture du sol et donc de la morphologie de la plage due aux tas de coquilles qui y sont déposés (photo 9);

- rareté de certaines espèces de poissons (tilapia) qui serait due à l'apparition des arches.

\footnotetext{
${ }^{9}$ Une durée d'arrêt de collecte permettant la reproduction des coquillages.
} 


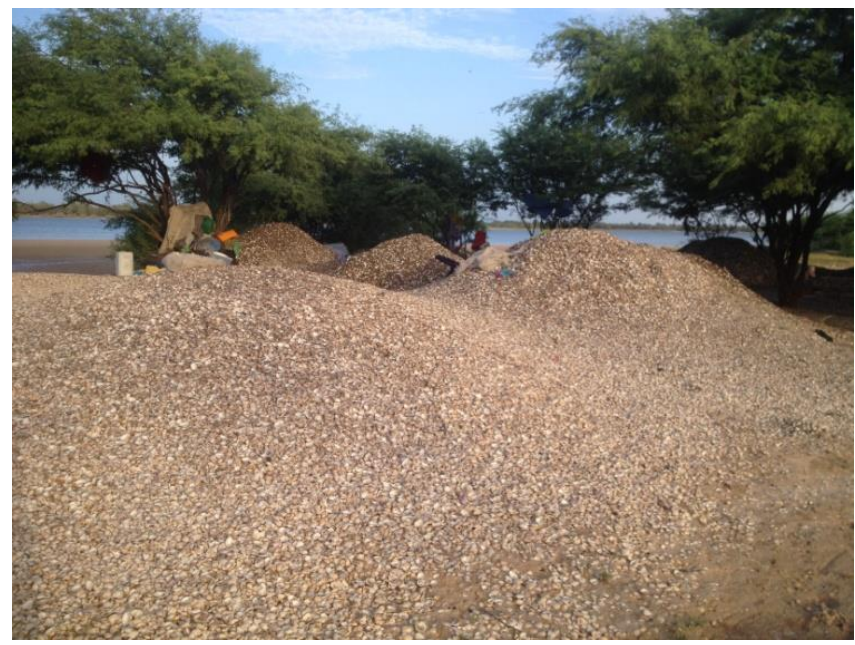

Photo 9 : Dépôt de coquille

\section{Discussion et conclusion}

L'ouverture de la brèche sur la Langue de Barbarie, en modifiant les conditions écologiques de l'espace gandiolais (Sy, 2013, Niang, 2017, Niang et $a l, 2020)$, a occasionné l'apparition et le développement de certaines espèces de coquillages dont les arches et les huîtres. L'exploitation des arches est devenue, dans un contexte de dégradation de l'activité agricole due à la salinisation des terres (Niang et al., 2020), la principale activité génératrice de revenus des populations de Mouit, de Moumbaye et de Dégou Niaye.

Cette recherche a révélé que les revenus obtenus de la collecte des arches ont permis de faire face aux besoins quotidiens et d'améliorer leur cadre de vie. Cependant, II est difficile d'obtenir des réponses précises sur certaines questions pour plusieurs raisons : les unités (de volume ou de poids) sont mal définies, les chiffres donnés concernent tantôt les coquilles fraîches, tantôt la chair séchée, ils se rapportent à un travail individuel ou de groupe par exemple une mère et ses filles, ils dépendent de la présence d'une pirogue qui permet de ramener en une fois les collectes importantes, enfin ils varient selon les saisons, les endroits, l'ardeur au travail, le temps passé, etc. En plus, des difficultés énormes ont été notées dans l'activité de collecte et occasionnent par ailleurs une entrave à l'accès de la ressource dans certains secteurs.

Dès lors, cette contribution sur l'exploitation des coquillages dans le Gandiolais s'harmonise avec l'enquête sur la collecte des arches dans le BasSaloum (Descamps, 1989). Celle conduite dans le delta du Saloum avait aussi procédé par questionnaire (18 informateurs ou groupe d'informateurs habitant les villages du Bas-Saloum ou ayant émigré dans les villes) et observation directe en accompagnant, sur le terrain un groupe de femmes du village de Niodior. Les questions étaient réparties sous cinq têtes de chapitres (Pratique de la collecte, expérience de la collecte, préparation des arches, contexte socio- 
économique, enfin perception des amas coquilliers anciens). Cette enquête avait permis alors de dégager les aspects technologiques, sociologiques et économiques d'une activité directement liée, dans le secteur des Rivières du Sud (Diop, 1990), à la présence de la mangrove. L'étude s'harmonise aussi avec celle portant sur l'écologie de 4 espèces exploitées dans les îles du Saloum (Anadara senilis, Crassostrea gasar, Murex duplex et Pugilina morio). Cette étude menée par l'IRD et la FIBA en 2011 avait pour objectifs d'apporter les bases biologiques scientifiques destinées à contribuer à l'exploitation durable de ces ressources. Elle a permis de comprendre la biologie de ces espèces afin d'élaborer un plan de gestion pour l'exploitation durable de ces coquillages pour le compte des femmes de ces îles.

En définitive, si au Bas delta du Saloum, on note une évolution notable du rôle des arches dans l'économie locale (auparavant, les populations pratiquaient la collecte essentiellement pour se nourrir, soit directement soit par troc; actuellement, elles collectent pour gagner de l'argent), dans le secteur du gandiolais, cette nouvelle activité centrée sur les arches est pratiquée dans un but essentiellement lucratif. Ainsi, le bénéfice est variable en fonction de l'abondance de la collecte et du prix de vente (en rapport avec l'offre et la demande). C'est la chair séchée qui est commercialisée ; seuls les enfants vendent parfois des arches en coque, qui doivent être alors rapidement préparées.

Les coquillages ne sont collectés pas seulement pour être consommés ou échangés contre de la nourriture, mais aussi pour être vendus et procurer un revenu dont la femme disposera à sa guise. Cette nouvelle option a été amplifiée par le fait que la coquille, autrefois délaissée, devient une source de gains : elle est en quelque sorte « recyclée ». Ainsi, les informations collectées et les observations faites sur le terrain ont fait ressortir beaucoup de manquements et de lacunes dans l'organisation des différents acteurs et la gestion de la ressource. C'est pourquoi, quelques recommandations susceptibles d'améliorer l'exploitation ont été formulées et concernent surtout le plan sanitaire. Il s'agit d'appuyer les exploitants sur le plan technique notamment la formation sur les techniques de collecte, de séchage et de labélisation des produits qui respecte les normes sanitaires. Pour cela, il leur faut une dotation de matériels de travail adéquat. Ainsi, au regard des manquements notés tant sur le plan organisationnel, socio-économique qu'environnemental il s'avère nécessaire d'encadrer les exploitants afin d'avoir une gestion durable de cette ressource renouvelable. Il serait aussi intéressant de faire un suivi spatio-temporel des teneurs de certains éléments métalliques (cadmium, plomb et mercure) d'Anadara senilis afin de connaitre les contaminations de cette espèce par ces métaux lourds et prévenir les exploitants. 


\section{References:}

1. ANDS, 2013. Recensement général de la population et de l'habitat, de l'agriculture et de l'élevage, république du Sénégal, Ministère de l'économie, des finances et du plan, $418 \mathrm{p}$.

2. DESCAMPS C. 1989. La collecte des arches, une activité bimillénaire dans le Bas-Saloum (Sénégal), enquête de terrain sur la collecte des arches, $07 \mathrm{p}$.

3. Diallo M. 2006. Aménagements hydrauliques et mutations du Bas estuaire du fleuve Sénégal : Impacts sur la nappe des sables dunaires du Gandiolais, Mémoire de DEA, UCAD, $79 \mathrm{p}$.

4. Diop E. S., 1990. La cote ouest-africaine du Saloum (Sénégal) à la Méllacoré (rep. De Guinée), thèse de Doctorat, ORSTOM, 366 p.

5. Diouf M. 2009. Guide de suivi bioécologique des coquillages exploites dans les iles de niodior, dionewar, falia et de fadiouth, rapport d'étude, $16 \mathrm{p}$.

6. GUEYE I. 2010. Evolution géomorphologique du littoral nord sénégalais (secteur du Gandiolais), analyse de la dégradation des ressources et perspectives de conservation, mémoire de Master, UGB, $147 \mathrm{p}$.

7. Jacoutot A. 2006. Modifications environnementales et conséquences dans le bas-delta du fleuve Sénégal. Cas du Gandiolais et du Parc National du Diawling, Master 2 EGEPM, 174 p.

8. MOSCAROLA J. 1989. Enquête et analyse de données Initiation à l'analyse de données, utilisations pédagogiques du sphinx, de l'enseignement à la recherche, première partie, $17 \mathrm{p}$.

9. Niang S. 2017. Dégradation chimique et mécanique des terres agricoles du Gandiolais (littoral Nord du Sénégal), analyse des dynamiques actuelles d'adaptation, Thèse de Doctorat unique, UGB, section géographie, laboratoire Leidy, $355 \mathrm{p}$.

10. Niang S. Sy A. A., Sow, S. Sy B. A. 2020. Analyse Par Approche Hydrogéochimique De La Distribution Spatiale De La Salinité Des Eaux De La Nappe Et Des Sables Quaternaires Du Secteur Des Niayes Du Gandiolais (Littoral Nord Sénégalais), European Scientific Journal August 2020 édition Vol.16, No.24 ISSN : 1857-7881 (Print) e - ISSN 18577431

11. Paillé, 2006. Méthode quantitative. Posture de recherche et travail de terrain, Paris, Armand Collin, $235 \mathrm{p}$.

12. RAP-LEMAR, 2011. Convention de recherche entre l'IRD et la FIBA en vue de la mise en place d'un plan de gestion pour l'exploitation durable des coquillages des îles du Saloum - Programme "Femmes et Coquillages", volet Recherche/Écologie. Rapport final de convention avec la FIBA. IRD Dakar, Sénégal. 38 p. 
13. SOW El., Ba T. 2019. Évolution de la Mangrove de la Réserve de Biosphère du Delta du Saloum, Sénégal, European Scientific Journal May 2019, édition Vol.15, No.15 ISSN : 1857 - 7881

14. Sow El, 2015. Le rôle des organisations non gouvernementales et des populations locales dans la politique de restauration de l'écosystème mangrove de l'estuaire du Saloum au Sénégal, mémoire de Master 2, UGB, section géographie, $102 \mathrm{p}$.

15. SY A. A., 2013. Dynamique sédimentaire et risques actuels dans l'axe Saint-Louis-Gandiol, littoral Nord du Sénégal, Thèse de Doctorat, UGB, $293 \mathrm{p}$.

16. SY B. A. et SY A. A. 2010. « Dynamique actuelle du cordon littoral de la grande côte sénégalaise de Saint-Louis à Niayam et ses conséquences » in Revue de géographie du Laboratoire Leïdi, UGB, no $8, p: 197-214$. 